\title{
Depression in Women: Implications for Health Care Research
}

\author{
Myrna M. Weissman* and Mark Olfson
}

Epidemiologic data from around the world demonstrate that major depression is approximately twice as common in women than men and that its first onset peaks during the childbearing years. Progress has been made in understanding the epidemiology of depression and in developing effective treatments. Much remains to be learned about the basic pathogenesis of depression and the specific treatment needs of depressed women and their offspring, especially during the reproductive years.

Nearly all writers upon insanity describe the mental derangements occurring during pregnancy, the puerperium, and the nursing period under the collective title "puerperal insanity.". . . Prolonged or excessive lactation is given as the chief cause. . . In most cases this is probably true, yet there are some cases in which the disease must be attributed to other etiological factors. . . . It appears .. . that the local (pelvic) irritations acting upon the central organ (brain) are active, both as determining the duration as well as the course of the mental disorder.

-From "Lactational Insanity," presented at the 44th annual meeting of the American Medical Association, 1893 (1)

Although current research has come a long way from the thinking expressed at the 1893 annual meeting of the American Medical Association (1), many gaps remain in understanding mental disorders. The conventional wisdom, even 30 years ago, was that depression was a disorder primarily of menopausal women and rarely occurred in children or adolescents. Recent epidemiologic studies have revised this view. It is now clear that depression is more common in women than in men across diverse cultures and is associated with substantial work (2-4) and family impairment (5) and high health care expenditures (2). The age of first onset is often younger than believed 30 years ago. Furthermore, depression affects women predominantly in their childbearing and child-rearing years. This epidemiologic finding has implications for treatment and research for improving women's health.

\section{Diagnosis and Epidemiology}

Over the last two decades, specific diagnostic criteria have been developed to define clinical depression (6). This specification has increased the reliability of the diagnosis and facilitated the conduct of systematic research. Major depression is the most common among a class of mood disorders. The essential features of major depression are low mood or a loss of interest or pleasure in usual

The authors are at the College of Physicians and Surgeons of Columbia University, New York, NY 10032, USA. M. Weissman is also at the School of Public Health and Division of Clinical and Genetic Epidemiology, New York State Psychiatric Institute, Unit 14, 722 West 168th Street, New York, NY 10032, USA. E-mail: weissman@child.cpmc.columbia.edu

*To whom correspondence should be addressed. activities. The disturbance is prominent, persistent, and associated with impaired functioning and a variety of other symptoms, including appetite and sleep disturbance, change in weight, psychomotor agitation or retardation, decreased energy, feelings of worthlessness or guilt, difficulty concentrating, and thoughts of death and suicide.

There is now considerable information on the rates of major depression based on epidemiologic studies conducted in the 1980s with comparable methods and diagnosis (6) from quite diverse cultures (7). Epidemiologic studies conducted in the United States (8), Canada (9), Puerto Rico (10), Paris (11), West Germany (12), Florence, Italy (13), Beirut, Lebanon (14), Korea (15), and New Zealand (16) all show convincingly that rates of depression are higher in women than in men, with about a twofold difference on average (7) (Fig. 1). Although the rates of depression vary by country, the predominance of women with depression is consistent across these different cultures. This

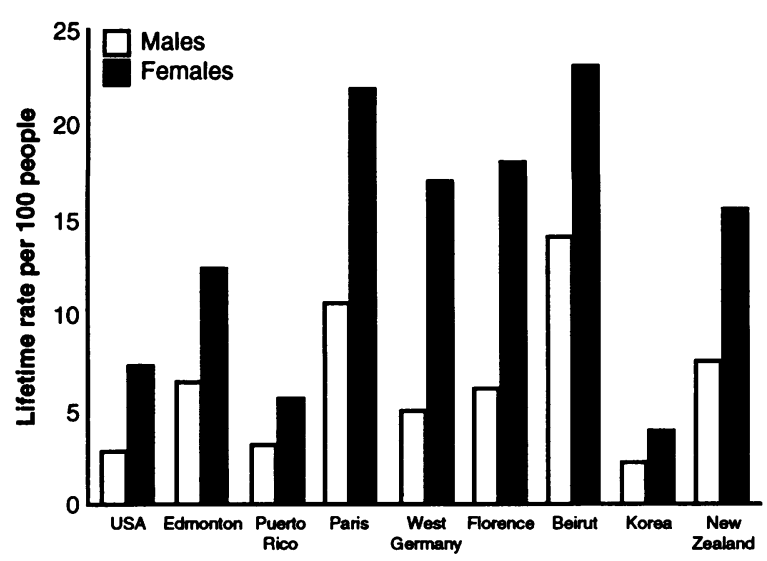

Fig. 1. Lifetime rate per 100 people for major depression (DSM-III) based on epidemiologic studies using the Diagnostic Interview Schedule (DIS) (20). The rates of major depression at each site were standardized to the age and sex distribution of the U.S. five-site household samples. Persons ages 18 to 64 are included in each site but West Germany, where ages 26 to 64 were included. The figure is adapted from (59), with permission. 
care and nutrition, disturbed sleep, lack of prenatal care, increased exposure to alcohol and drugs, and risk of suicide (25).

The U.S. Food and Drug Administration (FDA) carries warnings against the use of all psychotropic drugs during pregnancy. The effect of these medications on the human fetus cannot be investigated during premarketing experimental studies (26). Thus, data on abnormalities in fetal behavior and neurodevelopment must be based on observations of pregnant women who have inadvertently taken antidepressants or other psychotropic drugs (27-30). No published studies provide information on the long-term effects of in utero exposure to psychotropic drugs. Available data on early developmental milestones (lifting head, smiling, crawling, standing, talking, and walking) remain limited (31). Because many depressed patients smoke or abuse drugs, it is also difficult to distinguish the effects on the fetus of antidepressants from the effects of these other substances (32). Schatzberg and Cole (33) have summarized the situation quite well: "There is no clear evidence that any standard psychiatric drug does (or does not) cause birth defects."

Only scant information is available on dosing requirements in pregnancy for psychotropic drugs (34). Dosing needs may change during pregnancy because of decreased protein binding capacity, enhanced hepatic metabolism, progesterone-induced decrease in gastrointestinal motility, and decreased absorption (35). In one study that examined antidepressant dosing during pregnancy, patients required significantly increased antidepressant dosages in the final trimester (34).

Psychotherapy is a recommended alternative to medication for depressed patients during pregnancy $(25,36)$. Although there is a considerable body of evidence supporting the efficacy of psychotropic drugs in reducing acute depressive symptoms, comparatively few studies have included psychotherapy. In some studies, medications and time-limited psychotherapies, developed specifically for major depression, had equal efficacy in reducing depressive symptoms (37-39). In general, drugs for the treatment of depression have a faster and more consistent onset of action than psychotherapy. Maintenance drugs and psychotherapy prevent the recurrence of depressive symptoms $(39,40)$. Relatively high maintenance doses of drugs are required for maximum efficacy (40). Maintenance medication over 3 years to prevent recurrence in patients who have had severe recurrent depression has been effective (40). In the same study, monthly interpersonal psychotherapy prevented recurrences in nearly $70 \%$ of the patients over a 36-week period (40). If these results could be generalized to depressed pregnant women, psychotherapy could provide an effective alternative to antidepressant medications during gestation. Insurance policies and health care benefit packages that restrict access to psychotherapy may be detrimental to the health of women who require treatment for depression during pregnancy and the postpartum period and who cannot take medication.

\section{Postpartum Period}

Epidemiologic studies show that women have an increased frequency of all types of psychiatric illness in the postpartum period (20). The rise is most dramatic within 30 days after childbirth and persists for up to 2 years (41). Three types of puerperal disorders have been described: postpartum blues, postpartum psychosis, and postpartum depression $(42,43)$.

Postpartum blues refer to the transient period of depression experienced by most women in the early postpartum period. Symptoms usually peak at approximately the fifth postpartum day and rapidly diminish thereafter (44). This syndrome may represent a variation of normal emotional changes after childbirth and is usually not treated. Postpartum blues are thought to be secondary to the fluctuation in hormone levels after childbirth. Observed biological correlates of postpartum blues include the absence of the expected postpartum increase in plasma tryptophan, altered platelet monoamine oxidase activity, and higher serum-bound cortisol levels (20).

Postpartum psychosis is a severe psychotic syndrome that is estimated to occur after 1.1 to 4 of every 1000 deliveries. More than half of the affected women meet diagnostic criteria for major depression. The onset is usually within the first 2 weeks after delivery, but the risk remains relatively high for the first 3 months postpartum. Many women with postpartum psychosis have symptoms resembling an organic brain syndrome such as confusion, attention deficits, distractibility, and clouding of the senses. Risk factors for postpartum psychosis include a prior history of psychiatric illness, bipolar disorder (45-47), and a family history of a major psychiatric illness. Although the cause of postpartum psychosis remains unknown (48), some researchers believe that low estrogen levels postpartum may precipitate psychosis through a supersensitization of central dopamine receptors (49).

In terms of severity, postpartum depression represents the midway point between mild blues and severe psychoses. Postpartum major depression, which often resembles other forms of major depression, occurs in 10 to $15 \%$ of new mothers. Although most such depressive episodes begin within 2 weeks of delivery, depression can occur at any time during the following several months (49). Psychosocial stressors and previous psychiatric history seem to be important risk factors $(45,49)$. Approximately 20 to $30 \%$ of preg- nant women with a prior history of depression will develop postpartum depression. Once a woman has suffered a postpartum depression, the risk of another episode with subsequent pregnancies approaches 50\%. Large shifts in progesterone, estrogen, cortisol, and beta-endorphin levels may contribute to postpartum depression (20).

Discontinuing maintenance antidepressant treatment during pregnancy places patients with recurrent depression at high risk of recurrence after delivery. Because antidepressants are secreted into breast milk, reinstituting antidepressants in the postpartum period poses a problem for nursing women. The long-term effects on the infant of antidepressants in breast milk have not been studied, and little information exists on their short-term effects (29). In the absence of these data, women who require antidepressants are usually advised to discontinue nursing (50). In some cases, this medical advice runs counter to strong social expectations that mothers nurse their babies and thus may be a source of guilt for the mother.

\section{Implications for Research}

Many gaps remain in our understanding of depression in women. We lack a coherent explanation of why the rates of major depression are higher in women than in men and why the rates begin to rise in females around puberty. The consistency with which these findings emerge suggests that biologic factors, such as endocrinologic and genetic influences, may contribute to the relative excess of depression in women. However, it is conceivable that there are universal social factors that depress women and account for the cross-cultural predominance of depression in women.

Because depression is primarily a disorder of women of childbearing age and because there are excellent treatments for depression, more research needs to be conducted on the short- and long-term effects on the developing fetus of in utero exposure to antidepressants. For ethical reasons, these studies need to be conducted on pregnant and nursing women who are inadvertently, rather than experimentally, exposed to antidepressants. Studies are needed that track the developmental milestones of infants exposed to antidepressants at various stages of prenatal and postnatal development. A major challenge of this research will be to sort out the effects of other drug and alcohol use from the effects of antidepressants.

Further study is also needed of antidepressant dose requirements during pregnancy, lactation, and over the menstrual cycle. Analogous studies need to be conducted on the effectiveness of psychotherapy. At the same time, methods are needed to reliably distinguish depressed pregnant women who 
can be safely managed with psychotherapy alone from those who require maintenance antidepressants.

Although postpartum blues, psychosis, and depression have been described for over a century, their etiology remains largely obscure. Studies of adopted children where the adopting mother has not undergone a pregnancy may help to sort out social and cognitive factors from biological factors in the development of depression. Endocrine studies that compare postpartum mothers who do or do not develop postpartum depressive syndromes represent a second approach.

Women of childbearing age have historically been excluded from the early stages of drug development primarily out of concern for the effects on fetuses. The FDA has recently ended its ban on permitting women to enter drug trials and has required drug companies to include more women in early clinical trials. As this policy takes effect, guidelines ensuring adequate contraception will be needed to safeguard against fetal exposure to psychotropic drugs (51).

Little information exists on sex differences in the effectiveness of antidepressants for patients at any age. Some data suggest that women may be less responsive than men to tricyclic antidepressants $(52,53)$. One explanation for sex disparities in antidepressant responsiveness involves sex differences in the bioavailability of antidepressants (35, 54). Important differences between women and men have been described in drug absorption, volume of distribution, hepatic metabolism, body weight, total blood volume, percentage of body fat, renal clearance, and a variety of other physiologic variables that may affect drug bioavailability (35). Careful studies are needed that compare plasma antidepressant levels in women and men administered comparable test doses.

Although a range of excellent treatments for depression is available, most depressed adults in the United States receive no treatment for their symptoms during the course of a year (55). In this context, the concentration of depression among females of childbearing age raises important issues for health services research. These include describing gender differences in the act of seeking health care (56), access to care, and the effectiveness of the various treatments. Although gender bias in the provision of appropriate treatment of cardiovascular disease has been documented (57), comparable studies for depression are rare (58).

Several cultural-economic factors also come into play in such gender bias. Women are more likely than men to be covered as dependents, to work part-time, or to work for small businesses that do not provide insurance. They are, therefore, more susceptible to disruption of coverage through divorce, death, and job loss. Studies are needed that evaluate the relation between health care insurance, gender, and outcome of depression.

Pregnancy provides medical opportunities for the detection and management of depression. Although a substantial body of literature describes the management of depression by internists and family practitioners, less attention has been devoted to the role of obstetricians and gynecologists. Research is needed that examines how effective these medical specialists are in managing their patients' depression.

In addition, the serious impact of maternal depression on offspring function has been well described (5). But we lack intervention studies to determine whether reducing maternal depressive symptoms with pharmacotherapy or psychotherapy improves offspring outcome. In fact, no studies have followed the course of depression in mothers to determine the precise effects of maternal depression on offspring.

We have come a long way since 1893 , when local pelvic irritations on the brain were considered an explanation for postpartum depression (1). Considerable progress has been made in understanding the epidemiology of depression and in developing effective and well-tolerated treatments. But much remains to be learned about the basic pathogenesis of depression and the specific treatment needs of depressed women and their offspring, especially during the reproductive years.

\section{REFERENCES AND NOTES}

1. G. H. Rohé, J. Am. Med. Assoc. 21, 325 (1893), reprinted in ibid. 270, 1180 (1993).

2. G. Simon, J. Ormel, M. Von Korf, W. Barlow, Am. J. Psychiatry 152, 352 (1995).

3. K. B. Wells, M. A. Burnam, W. Rogers, R. Hays, P. Camp, Arch. Gen. Psychiatry 49, 788 (1992).

4. W. E. Broadhead, D. G. Blazer, L. K. George, C. K. Tse, J. Am. Med. Assoc. 264, 2524 (1990).

5. M. M. Weissman et al., Arch. Gen. Psychiatry 44, 847 (1987).

6. Diagnostic and Statistical Manual of Mental Disorders. (American Psychiatric Association Committee on Nomenclature and Statistics, Washington, DC, ed. 3,1980 ).

7. Cross-National Collaborative Group, J. Am. Med. Assoc. 268, 3098 (1992).

8. L. N. Robins and D. A. Regier, Eds., Psychiatric Disorders in America: The Epidemiologic Catchment Area Study (Free Press, New York, 1991).

9. H. S. Orn, S. C. Newman, R. C. Bland, Acta Psychiatr. Scand. Suppl. 77 (338), 17 (1988).

10. G. J. Canino et al., Arch. Gen. Psychiatry 44, 727 (1987).

11. J. P. Lepine et al., Psychiatr. Psychobiol. 4, 267 (1989).

12. H. U. Wittchen, C. A. Essau, D. von Zerssen, J. C. Krieg, M. Zaudig, Eur. Arch. Psych. Clin. Neurosci. 241, 247 (1991).

13. C. Faravelli, B. G. Degl'Innocenti, L. Aiazzi, G. Incerpi, S. Pallanti, J. Affective Disord. 20, 135 (1990).

14. E. Karam, paper presented at a meeting of the International Traumatic Stress Society, Washington, DC 26 October 1991.

15. C. K. Lee et al., J. Nerv. Ment. Dis. 178, 242 (1990).

16. J. E. Wells, J. A. Bushnell, A. R. Hornblow, P. R. Joyce, M. A. Oakley-Browne, Aust. N. Z. J. Psychiatry 23, 315 (1989).

17. R. C. Kessler et al., Arch. Gen. Psychiatry 51, 8 (1994).

18. N. Breslau, L. Schultz, E. Peterson, Psychiatry Res., in press.
19. J. K. Sargeant, M. L. Bruce, L. P. Florio, M. M Weissman, Arch. Gen. Psychiatry 47, 519 (1990).

20. S. I. Wolk and M. M. Weissman, in Review of Psychiatry, J. Oldham and M. Riba, Eds. (American Psychiatric Press, Washington, DC, 1995), vol. 14, chap. 9

21. I. H. Gotlib, V. E. Whiffen, J. H. Mount, K. Milne, N. I. Cordy, J. Consult. Clin. Psychol. 57(2), 269 (1989).

22. T. Kitamura, S. Shima, M. Sugawara, M. A. Toda, Psychol. Med. 23, 967 (1993)

23. R. Kumar, M. Marks, A. Weich, Prog. Neuro-Psychopharmacol. Biol. Psychiatry 17, 571 (1993).

24. S. A. Montgomery, in Psychopharmacology: The Fourth Generation of Progress, J. E. Bloom and D. J. Kupfer, Eds. (Raven Press, New York, 1995), chap. 89, pp. 1043-1052.

25. American Psychiatric Association Work Group on Major Depressive Disorder, Am. J. Psychiatry 150, 1 (1993).

26. L. J. Miller, Psychiatr. Med. 9, 275 (1991).

27. L. L. Kerns, J. Nerv. Ment. Dis. 174(11), 652 (1986)

28. A. Pastuszak et al., J. Am. Med. Assoc. 269, 2246 (1993).

29. L. S. Cohen, Curr. Affect. Illness 11, 5 (1992).

30. J. A. Baldwin, E. J. Davidson, A. L. Pritchard, J. E. Ridings, Acta Psychiatr. Scand. Suppl. 80 (380), 37 (1988).

31. G. Viggedal, B. S. Hagberg, L. Laegreid, M. Aronsson, J. Child Psychol. Psychiatry Allied Discip. 34 (3), 295 (1993).

32. M. J. Tueth, J. Am. Med. Assoc. 270 (18), 2177 (1993).

33. A. F. Schatzberg and J. O. Cole, Manual of Clinical Psychopharmacology (American Psychiatric Press, Washington, DC, 1991), p. 315.

34. K. L. Wisner, J. M. Perel, S. B. Wheeler, Am. J. Psychiatry 150, 1541 (1993).

35. K. A. Yonkers and J. A. Hamilton, in Review of Psychiatry, J. Oldham and M. Riba, Eds. (American Psychiatric Press, Washington, DC, 1995), vol. 2, pp. 307-332.

36. Agency for Health Care Policy and Research, Technical Report 93-0551 (U.S. Department of Health and Human Services, Rockville, MD, 1993).

37. I. Elkin et al., Arch. Gen. Psychiatry 46 (11), 971 (1989).

38. G. L. Klerman, A. DiMascio, M. M. Weissman, B. Pru soff, E. S. Paykel, Am. J. Psychiatry, 131, 186 (1974).

39. A. DiMascio et al., Arch. Gen. Psychiatry 36, 1450 (1979).

40. E. Frank et al., ibid. 47, 1093 (1990).

41. R. E. Kendell, J. C. Chaimers, C. Platz, Br. J. Psychiatry 150, 662 (1987).

42. M. J. Garvey and G. D. Tollefson, J. Reprod. Med. 29, 113 (1984)

43. B. Zuckerman, H. Bauchner, S. Parker, H. Cabral, J. Dev. Behav. Pediatr. 11, 190 (1990).

44. M. W. O'Hara, J. A. Schlechte, D. A. Lewis, E. J. Wright, Arch. Gen. Psychiatry 48, 801 (1991)

45. I. F. Brockington, C. Martin, G. W. Brown, D. Gold berg, F. Margison, Br. J. Psychiatry 157, 331 (1990)

46. J. Davidson and E. Robertson, Acta Psychiatr. Scand. 71, 451 (1985).

47. R. E. Kendell, J. Psychosom. Res. 29, 3 (1985).

48. D. Dowlatshahi and E. S. Paykel, Psychol. Med. 20 655 (1990)

49. C. J. Martin, G. W. Brown, D. P. Goldberg, I. F Brockington, J. Affective Disord. 16, 283 (1989).

50. J. F. Mortola, Psychiatr. Clin. N. Am. 12, 69 (1989)

51. A. C. Mastroianni, R. Faden, D. Federman, Eds., Women and Health Research: Ethical and Legal Is sues of Including Women in Clinical Studies (National Academy Press, Washington, DC, 1994), vol. 1.

52. A. H. Glassman, J. M. Perel, M. Shostak, S. J. Kantor, J. L. Fleiss, Arch. Gen. Psychiatry 34, 197 (1977).

53. E. Frank, L. L. Carpenter, D. J. Kupfer, Am. J. Psychiatry 145, 41 (1988).

54. K. A. Yonkers, J. C. Kando, J. O. Cole, S. Blumenthal, ibid. 149, 587 (1992).

55. D. A. Regier et al. , Arch. Gen. Psychiatry 50, 85 (1993).

56. R. C. Kessler, R. L. Brown, C. L. Broman, J. Health Soc. Behav. 22, 49 (1981).

57. J. N. Tobin et al., Ann. Intern. Med. 107, 19 (1987).

58. A. A. Hohmann, Med. Care 27, 478 (1989).

59. M. M. Weissman et al., World Psych. Assoc. Teaching Bull. Depression 2, 1 (1994).

60. This work was supported in part by grants $\mathrm{MH}-37592,-36197$, and -50666 from $\mathrm{NIH}$. 